Revista de Psicología de la PUCP. Vol. XVI, 1, 1998.

\title{
LA PSICOLOGÍA INFANTIL AL INICIO DEL SIGLO XXI. IMPACTO DE FACTORES IDEOLÓGICO/ CULTURALES EN NORTEAMÉRICA. REPERCUSIONES PARA EL PERÚ
}

\author{
Martha Lequerica ${ }^{1}$ \\ Montclair State University
}

En este artículo se analiza el impacto de tres factores ideológico/culturales en el avance y desarrollo de la Psicología Infantil en Norteamérica durante las últimas cuatro décadas. E1 Movimiento de Derechos Civiles, el feminismo y la lucha contra la pobreza han tenido una influencia decisiva en la concepción, crianza y educación del niño, asi como en la medición de la inteligencia, el emplazamiento en clases especiales y el ímpetu por la educación preescolar y la propagación del bilingüismo como medio de enseñanza. Se plantea y recomienda la necesidad de aplicar el mismo análisis al estudio de la psicología infantil latinoamericana $y$ en específico a la del Perú.

Palabras claves: psicologia infantil, derechos civiles, crianza, desarrollo, medición de la inteligencia.

The child psychology at the beginning of the twenty first century. Impact of the ideological/cultural factors in North America. Repercussions in Peru

The purpose of this paper is to analize the impact the Civil Rights Movement, feminism and the War Against Poverty have had on the evolution and reshaping of child psychology in the Linited States, during the last four decades. Thesc threc ideological/cultural factors have played a major role in child/rearing, education and conceptualization of the child's moral and sex role development as well as in intelligence measurement/assessment, placement in special classes and the dissemination of bilingualism as a tool for teaching. How to apply a similar analysis to the study of child psychology in Latin America and more specifically in Perú, is proposed and recommended.

Key words: Child psychology, civil rights, rearing, development, intelligence measurement.

1. Doctora en Psicología y Profesora Asistente de Psicologia del Departamento de Psicología de la Universidad Estatal de Montclair. Autora de Artículos sobre la Psicología del Niño y la Evaluación. Dirección: Department of Psychology. Upper Montclair, New Jersey 07046. EUA. Fax: 7186017294. 

La psicología infantil en Norteamérica ha experimentado cambios profundos en las últimas tres a cuatro décadas, o desde 1960 en adelante. Desde una posición estable y delineada claramente por modelos teóricos de personalidad, desarrollo cognitivo y psicosocial heredados de Freud (1920/1950, en Freud, 1952), Piaget (1954; 1969), Erikson (1963), y los conductistas (Skinner, 1951,), la psicología infantil se ha transformado casi súbitamente desde la década del sesenta en adelante, en una área resonante de cambios y ampliación de sus fronteras, en constante proceso de revisión y expansión. La confluencia y acción interactiva de varios factores ha contribuido a estos cambios fundamentales y a la nueva dirección.

\section{Influencias}

Un grupo de factores influyentes en el cambio de la psicología infantil son de naturaleza ideológico/cultural. Por ejemplo, el movimiento de derechos por igualdad civil de la raza negra, la lucha contra la pobreza y el feminismo, han tenido un impacto decisivo en la concepción del desarrollo del niño, su forma de crianza, la edad de intervención, entre otros.

Factores de indole sociodemográficos, constituyen otro grupo influyente. Por ejemplo: el aumento de hogares en que los dos padres trabajan, el incremento del divorcio, hogares encabezados solamente por la madre, el crecimiento agigantado de la inmigración, el surgimiento y expansión del abuso de drogas entre las mujeres, y el aumento acelerado del embarazo de adolescentes, cuentan en este grupo.

Otros factores de influencia en la expansión de la psicología infantil en Norteamérica son de tipo teórico/psicológico como el nacimiento de la psicología cognitiva (Miller, 1962; Neisser, 1967) y los modelos de procesos 
informativos (Atkinson y Shiffrin, 1977) aplicados al desarrollo infantil (Flavell, 1977; Kagan y Moss, 1983) así como la aplicación de los principios del espacio vital de Lewin (1951) a la formulación ecológica del desarrollo postulado por Bronfenbrenner en 1976.

Finalmente, de no menos importancia, son los factores legislativos emanados en la década del setenta en el esfuerzo de combatir la discriminación contra las minorías (Diana contra Board de Educación, 1970; Larry contra Riles, 1972) al proponer el uso del inglés y las pruebas estandarizadas en la evaluación de niños inmigrantes, así como el reconocimicnto de los derechos legales paternos en el proceso de evaluación de niños bilingües o marginados. Varias legislaciones estatales y federales también emanaron para establecer las pautas de intervención temprana con niños impedidos del desarrollo; por ejemplo, en el caso del autismo, parálisis cerebral o mongolismo (Ley Pública 94-142 de 1975 y sus apéndices o amendamientos de 1986 y el Acta para Individuos con Impedimentos de 1995 - IDEA).

En realidad todos estos factores, -sean demográficos, ideológico/ culturales, legislativos o teóricos- están vinculados y entrelazados intimamente, influyéndose y relacionándose recíprocamente, así como formando en esencia el contexto sociohistórico/cultural de la psicología como ciencia y disciplina. Por ejemplo, si analizamos los decretos legislativos contra la discriminación de minorías en la medición de inteligencia y emplazamiento en clases especiales, se observa que dicha legislación está influenciada por el movimiento ideológico de igualdad de derechos civiles, así como el incremento de las minorías como fuerza demográfica y de poder electoral político/legislativo. Similarmente, la expansión del feminismo y el retorno de la mujer en masa al mercado laboral y al estudio profesional (factor ideológico/cultural) que ha revolucionado el sistema de crianza de los niños, está muy vinculado al aumento del divorcio y de los hogares formados por mujeres solas (factor sociodemográfico).

El análisis de estos diferentes factores y su contribución es imperativo para comprender cómo se conceptualiza, se enseña y se practica la 
psicología infantil actualmente en los Estados Unidos. A partir de la década de 1980 en adelante, las corrientes teóricas y psicológicas se han estabilizado y aún más, se han integrado en lugar de continuar las luchas ideológicas de décadas anteriores. Y los avances jurídicos se han incorporado en el diario conducir de la psicología infantil en las escuelas, hospitales y clínicas privadas. El aspecto socio/demográfico por otra parte, continúa modificándose y cambiando constantemente debido a influencias socio/tecnológicas (como el de la inseminación artificial) o los cambios internos de la sociedad americana tales como la constante revolución sexual, la presencia del SIDA, y los cambios de estilo de vida (familias de homosexuales adoptando a niños) y nuevas concepciones en la organización de la familia (familias con hijastros, padrastros y nuevos hijos de parejas en segundo o tercer matrimonio, custodia dividida entre padre y madre después del divorcio, etc).

En este artículo se enfocará exclusivamente el aporte de los factores ideológico/culturales en el desarrollo, evolución y expansión de la psicología infantil en las últimas cuatro décadas y al aproximarse el siglo XXI. A continuación, bajo la sección de Reflexiones y Repercusiones, se incitará la discusión y planteamiento de un análisis semejante al estudio de la psicología infantil en América Latina y principalmente, en el Perú.

\section{Factores ideológico/culturales}

Se definen como estos a corrientes de pensamiento, filosofía y acción que promueven al individuo como miembro de una sociedad a iniciar, cambiar o reformar su planteamiento humano en relación a cuestiones esenciales, tales como el gobierno, los derechos humanos, el rol de la mujer en la sociedad y en la educación de los niños, entre otros.

En los últimos cuarenta años, varias concepciones ideológicas o culturales han tenido un impacto categórico en el desarrollo y rumbo de la psicología infantil de Norteamérica. Entre ellas sobresalen la lucha por los derechos civiles del grupo afroamericano (Movimiento de Derechos Civiles), la liberación sexual y laboral de la mujer en la sociedad (femi- 
nismo), y la lucha contra la pobreza (Guerra contra la Pobreza). Se analizará cada uno de estos factores ideológico/culturales y su impacto en la crianza, educación, estudio del niño, asi como la práctica de la psicología y la terapia infantil en la actualidad y a los inicios del nuevo siglo.

\section{El movimiento de derechos civiles}

En las décadas de los cincuenta y sesenta, el movimiento por paridad de derechos con la mayoría blanca, dirigidos por líderes y ciudadanos de raza afroamericana se inició, expandió y obtuvo varias victorias legislativas y populares. Originado como fuerza contra la segregación en restaurantes, ómnibus y baños públicos, el movimiento se convirtió en una cruzada que abarcó todos los aspectos de la vida nacional. Una de sus victorias iniciales fue la abolición de la segregación racial en las escuelas con el pase del decreto Brown contra el Centro Educación de 1954 que estableció que la segregación racial es inberentemente desigual y anticonstitucional. Aunque implementado lenta y gradualmente, este decreto tuvo un impacto definitivo en la psicología infantil, fermentando entre varios corolarios, el famoso estudio de Coleman, Hoffer y Kilgore (1966) acerca del origen de las desigualdades raciales en el rendimiento escolar. Este informe delineó por primera vez el efecto del nivel socioeconómico en el rendimiento escolar de los niños y dio origen -entre otros factores- a que los educadores trataran de disminuir las desigualdades académicas por medio del enriquecimiento cultural, la intervención temprana o la educación compensatoria para favorecer el desarrollo cognitivo del niño de nivel socioeconómico bajo.

Otro corolario del informe Coleman fue la iniciación e incentiva del transporte de niños afroamericanos por el autobus de su vecindad a la escuela localizada en un barrio blanco (busing), con el objetivo de modificar el desequilibrio racial dentro de las escuelas y convertirlas en centros sin segregación. Mucha literatura emanó con respecto al resultado del busing y los esfuerzos por combatir la segregación en el rendimiento de los niños afroamericanos. Sin embargo, una revisión de esta literatura indica que 128 
el busing no dio los resultados esperados y no provocó el incremento académico de los niños minoritarios (Orfield, 1983; Rist, 1978; St. John, 1981).

El énfasis en el rendimiento escolar de las minorías y su superación académica originó también el debate y controversia acerca de la relación entre los factores de raza, clase social e inteligencia. Por ejemplo, niños de raza afroamericana (como grupo) que son marginados socialmente y viven en condiciones de vivienda de un nivel menor al estándar, aprenden muy poco y de manera semejante a niños inmigrantes/bilingües; rinden deficientemente en la escuela, leen en un nivel por debajo de su grado y abandonan temprano la escuela sin graduación debido a sus frustraciones académicas (Halsell Miranda y Santos de Barona, 1990). El debate acerca de las causas del rendimiento pobre y el bajo nivel de inteligencia obtenida mediante pruebas estandarizadas de inteligencia fue encabezado por el psicólogo Arthur Jensen que en 1969 publicó su artículo controversial Podemos elevar el CI y el rendimiento académico, en el cual propuso que las diferencias de CI entre los niños blancos y los afroamericanos se deben a diferencias genéticas de inteligencia que son heredadas y transmitidas del ambiente, por ejemplo, de la pobreza y el estrés. El artículo de Jensen provocó una discusión virulenta con defensores que calificaron sus argumentos de racistas. Aunque este debate se calmó en la década de los ochenta, reapareció en 1994, cuando Herrnstein y Murray publicaron otro libro controversial titulado La cuna Bell: Inteligencia y estructura social en la vida americana, en el que se repite el tema básico de Jensen con nuevos argumentos y recomendaciones.

Es también en la década de los setenta cuando estudios del efecto del bilingüismo en la inteligencia trataron de establecer si los efectos son positivos, negativos o neutrales. Esta controversia se inició en la década del cuarenta, siguiendo la primera masa de inmigrantes de origen hispano que inundó las costas norteamericanas. El estudio de Peal y Lambert (1962) estableció que los efectos del bilingüismo en la inteligencia son mediados por varios factores tales como el nivel socioeconómico de los niños y la familia y el prestigio de la lengua nativa. Las ganancias observadas 
en los niños bilingües comparados con los monolingües se dieron en tareas de creatividad o pensamiento divergente del niño (inteligencia creativa, no académica). El estudio de los diversos aspectos sociolingüísticos y socioemocionales del bilingüismo continúa y se ha propugnado cartas legislativas como el Acta de Educación Bilingüe y la creación de clases bilingües o donde se hable la lengua nativa de niños inmigrantes llegados de varias partes del mundo, como Persia, India, Egipto, Puerto Rico, Cuba, Sudamérica, entre otros.

El movimiento contra la discriminación racista favoreció el desarrollo de la cultura pluralista en Norteamérica. Desde los años setenta en adelante se ha fomentado oficialmente en las escuelas, universidades y establecimientos del gobierno la tolerancia y convivencia entre los grupos de inmigrantes o ciudadanos de varias razas que conservan su patrimonio lingüístico, racial y cultural en lugar de la asimilación forzada por la cual los inmigrantes renunciaban a su origen y herencia nativa en sus esfuerzos por aparecer y ser aceptados como norteamericanos puros. Esta tolerancia cultural y racial, heredada de la lucha por la universalidad de los derechos humanos, tomó otro impetu con la política del presidente Johnson en 1965 en su guerra contra la pobreza.

\section{La lucha contra la pobreza}

Sociológicamente, en los Estados Unidos, el nivel de pobreza se define en términos de privación absoluta (Robertson, 1987). En ese sentido, el gobierno establece el presupuesto anual debajo del cual una familia es considerada polve. En 1985 por ejemplo, el nivel de pobreza para una familia de 4 personas era $\$ 10,980$ o sea $\$ 211$ por semana para cubrir los gastos de vivienda, nutrición y vestuario asi como costos de salud (U.S. Census Bureau, 1986).

En los años de 1963 y 1965 alrededor de los cuales empezó la campaña oficial del gobierno contra la pobreza, el $18 \%$ de la población americana estaba viviendo debajo del nivel calificado como pobre. Debido a los programas organizados por el gobierno en forma intensiva, el 
porcentaje bajó considerable a 12 o $13 \%$ alrededor de 1973 y luego ha ascendido paulatinamente y se considera que ahora en la década del noventa, el nivel de pobreza se ha intensificado especialmente entre las familias presididas por mujeres solas y madres adolescentes (Committee for Economic Development, 1987). Actualmente New York Times, Sept.29, 1996) se ha registrado que $22 \%$ de niños americanos viven en pobreza, lo cual representa un porcentaje más alto que en los inicios de la guerra contra la pobreza en 1963.

Una de las armas más poderosas usadas por el gobierno para la erradicación de la pobreza desde la década del sesenta en adelante, fue la educación. El foco de la campaña educativa contra la pobreza se concentró en la creación de un programa preescolar de gran impacto hasta el momento actual. Fiste programa fundado bajo los auspicios del gobierno de Johnson y el patrocinio teórico del psicólogo Edward Zigler y el pediatra Howard Richmond, se llama Head Start (Zigler, 1991). Iniciado como un programa de verano por ocho semanas en 1965, se expandió y ramificó rápidamente, y actualmente después de 33 años, cuenta con centros en todas las áreas del país incluyendo las zonas migratorias, los campos de reservaciones indígenas, las áreas rurales de Ohio, las montañas de Apalachia y los centros de Chicago, Los Angeles y Nueva York.

El programa ofrece intervención de tipo comprensivo en los años preescolares. Niños de 3 a 5 años son recluidos en zonas marginales y registrados en el programa que ofrece una variedad muy amplia de servicios que incluyen educación, nutrición, servicios sociales, intervención médica, talleres de entrenamicnto para los padres en temas de crianza y desarrollo, así como evaluación y derivación de los niños a riesgo con problemas de lenguaje, aprendizaje, emocionales o de conducta.

Como parte de la asistencia social familiar del programa se brinda el escrutinio y soporte en citas de salud, con inmunizaciones, citas médicas y chequeos de salud. De gran importancia es la participación de los padres que ayudan como voluntarios en los salones de clases y en excursiones 
educativas así como en la administración de fondos y funcionamiento del programa.

La idea central es que los padres de familia constituyan un elemento activo en la educación y mejoramiento médico/social/educativo de sus niños, mientras también ellos son recipientes de entrenamiento educativo (clases de inglés, preparación del diploma de secundaria), así como entrenamiento de trabajo (en limpieza, secretariado de oficina, asistente de maestro o asistente social). Tales entrenamientos contribuyen activamente a que las familias de presupuesto marginal abandonen paulatinamente las filas de Asistencia Pública y se incorporen como miembros activos en la producción económica del país (Lequerica, 1994; Zigler, 1991)

La creación de Head Start en su concepción filosófica-educativa es una contribución muy poderosa a la psicologia preescolar norteamericana y posiblemente a la psicología preescolar global por lo siguiente: a) reconoce la posición desventajosa del niño pobre en relación a la escuela $y$ las ocupaciones futuras; b) usa el acercamiento comprensivo que incluye varios aspectos del desarrollo del niño (salud, alimentación, cuidado dental, buena vivienda), y no sólo la educación y el desarrollo cognitivo; c) reconoce y emplea la colaboración e intervención de los padres y su entrenamiento como básico elemento para el avance de las familias del estrato social desventajoso. Aunque las investigaciones realizadas para documentar los resultados de Head Start en el crecimiento cognitivo y rendimiento escolar han mostrado sólo avances a corto plazo, como el estudio Westinghouse (Cicirelli, 1969), otras investigaciones han dado resultados más positivos. Schweinhart (1985), en la investigación longitudinal emprendida en Ypsilanti, (Michigan), encontró menos porcentajes de desempleo, embarazo de adolescentes y un índice más bajo de la delincuencia entre los adultos que de niños asistieron a estos programas, que entre aquellos que no lo hicieron.

Con respecto a la psicología infantil, el programa de Head Start representa el esfuerzo más consistente y substancial en nuestro hemisferio, de reconocer las necesidades físicas y psicosociales de la niñez pobre, y de 
tratar de enfrentarlas a través de un programa subvencionado por el gobierno federal de Washington. Aunque en los años republicanos de Reagan hubo intentos de disminuir los fondos financieros, la subvención del gobierno se mantuvo constante, aunque no aumentó. Head Start como programa educativo/social y como filosofia de prevención ha inspirado varias investigaciones científicas, varios libros, así como conferencias nacionales, regionales y locales. Además, muchos artículos se han inspirado en la labor docente, social y psicológica realizada a través de los años y que continúan sólidamente hasta el presente (Lequerica, 1994a; 1994b; Zigler,1984; 1991).

A continuación se analizará otro de los factores ideológicos de gran influencia en la psicologia infantil.

\section{E1 feminismo}

Paralelo al movimiento de derechos por la igualdad civil, surgió el movimiento de liberación femenina auspiciado por el descubrimiento de la píldora contraceptiva así como el advenimiento de las publicaciones de Friedan Feminine Mystique (1963) y de Gloria Steinem (editora de la revista $\mathrm{Ms}$ ).

El movimiento feminista se centró básicamente en dos premisas: a) la liberación sexual y b) la liberación económica de la mujer a través del enfrentamiento contra roles tradicionales de ama de casa, excluída del rol profesional. La mujer americana de la década del treinta en adelante y en particular de los años cincuenta, siguiendo la segunda guerra mundial, era una mujer destinada al hogar, a tener niños y cuidar la casa, derivando su orgullo e identidad personal de su rol como esposa y madre.

Los hogares típicos norteamericanos en los años cincuenta se representaban en casas con una verja blanca y con el césped verde con los esposos dedicados al trabajo todos los días, mientras la esposa se quedaba en la casa con los niños y con la compañía del perro y un gato, como elementos clásicos del hogar suburbano y la vida familiar completa. Sin 
embargo el ímpetu por el logro de la mujer como ser humano que necesita realizar y desenvolver todas sus posibilidades (intelectuales, sociales, económicas, asi como físicas y biológicas), volcaron a la mujer de la placidez y a la vez monotonía del hogar, hacia la conquista de un puesto de avance en la medicina, el mundo académico, financiero y legislativo asi como en la exploración sexual, reproductiva y de igualdad con el hombre.

La revolución ideológica del feminismo descubrió a veces lentamente y otras violentamente la prevalencia en la sociedad moderna de una perspectiva singularmente masculina, también llamada sexista, y que estaba en control de la medicina, historia, arte, la psicología, y el desenvolvimiento de la vida cotidiana. La visión sexista de la sociedad y sus individuos en ella, ponía a la mujer no sólo en un plano distinto dictado por su biología pero indudablemente en un plano inferior, esterotípico y asfixiante. El psicoanálisis y las teorías de la personalidad formuladas por Freud $y$ seguidores fueron criticadas ávidamente y en su reemplazo una psicología feminista para la terapia y la explicación de la sexualidad femenina emergieron como corolarios de la nueva ideología feminista.

Asimismo se descubrió paulatinamente que la visión de supremacía masculina o sexista perjudica igualmente al hombre tanto como a la mujer porque el rol tradicional de padre como proveedor de familia envuelto exclusivamente en el trabajo de oficina/fábrica, fuera de casa y de los niños, priva al hombre de dar y recibir nutrimiento emocional. El resultado de esta división sexista de la sociedad es que ni el hombre ni la mujer reciben la oportunidad de desarrollarse como seres humanos integrales y completos; esta teoría fue expuesta brillantemente por Dinnerstein en La Sirena y el Minotauro: Arreglos Sexuales y la Indisposición (malaise) Humana, publicado en 1976.

Las más importantes repercusiones del feminismo en el campo de la psicología infantil son las siguientes:

Creación de un enfoque feminista del desarrollo bumano, como por ejemplo, el desarrollo moral de las niñas basado en diferencias con el sexo masculino 
(Gilligan, 1982), y no en la supremacía o concepción del modelo masculino como lo postularan Freud (1920\1952) o Kolhberg (1969).

El reconocimiento del sexismo en la educación escolar, que tradicionalmente asignara habilidades científicas y de matemáticas para los varones exclusivamente, mientras se identificaba a las niñas con las ciencias domésticas, literarias o artísticas. Esta segregación y diferenciación mantuvo al sexo femenino en la sociedad norteamericana alejado de partes integrales de la cultura tales como la medicina, ingeniería, arquitectura. El feminismo ha permitido el lento desenlace de esta arbitraria diferenciación dando ímpetu a la incursión de la mujer en áreas como la exploración astronaútica, la medicina, la academia, la ley.

La incorporación masiva de la mujer al trabajo fuera del hogar (como profesional, obrera, congresista, etc), originó formas alternativas de crianza y cuidado no dependientes directamente del cuidado materno a tiempo completo como se hiciera tradicionalmente. Este cambio en la disponibilidad de la mujer para la crianza de los niños, produjo las siguientes consecuencias:

- El surgimiento y difusión de centros de guardería o cunas para cuidados de niños mientras las madres están en el trabajo. Estas guarderías mantienen a los niños en locales autorizados con personal entrenado. La proliferación de estos centros durante la década del setenta en adelante produjo una plétora de estudios psicológicos en respuesta a preguntas tales como ¿Cuál es el efecto de las guarderías en el desarrollo emocional y cognitivo del niño?, y aún más crucial la pregunta que muchas madres se hacían al dejar a sus niños en estos centros: ¿Tienen las guarderías un efecto perjudicial en el desarrollo del niño?. El consenso de las investigaciones realizadas en las décadas que siguieron al movimiento feminista estableció lo siguiente: Los centros de cuna o guardería tienen resultados positivos en el campo cognitivo y del lenguaje siempre y cuando estén equipados con personal competente y ofrezcan un programa de alta calidad. Programas de calidad son aquellos que ofrecen bajas proporciones de niños a adultos, grupos 
pequeños de interacción, y cuidadores entrenados en el desarrollo cognitivo y socioemotivo del niño (Scarr, 1998).

La tarea crucial para los padres consiste en encontrar los programas de calidad pues muchos centros constituyen respuestas improvisadas a la necesidad masiva que surgió casi súbitamente con el retorno de la mujer/madre al trabajo. Los cánones de calidad se establecieron poco a poco y muchos niños y padres fueron víctimas de la falta de criterio establecido y de cierto caos que existiera como resultado de la improvisación y el apuro. Además, los centros de calidad son a menudo de alto precio y por lo tanto solamente accesibles a las familias profesionales y no a las de madres que trabajan en fábricas, oficinas o centros laborales manuales.

- El aumento de niños que cargan su propia llave (latchey key kid) y que son responsables de entrar a su casa solos después de la escuela (de $3 \mathrm{pm}$ a 6pm) donde esperan a sus padres en soledad o en compañía de la televisión y el teléfono, pero básicamente sin supervisión adulta. La difusión de este fenómeno de auto/cuidado de niños aún desde los 7 u 8 años, produjo otra ola de investigaciones y preocupación de los expertos acerca de los efectos en el desarrollo emocional del niño. Los resultados obtenidos por las investigaciones son mixtos. Algunos autores enfatizan el aumento del sentido de responsabilidad en el niño; la mayoría sin embargo deploran el riesgo al desarrollo infantil provocado por la ausencia de la supervisión adulta, y las consecuencias del uso indiscriminado de la televisión, el uso intensificado de cigarrillos, alcohol o marihuana en los adolescentes que permanecen solos (Riley, 1997).

- Laparticipación más activa del padre en el cuidado del infante y del niño preescolar. Los maridos y padres de familia tenían en la sociedad tradicional de la era posterior a la segunda guerra mundial un papel muy específico y limitado en la crianza y desarrollo de los niños: el de proveedor del dinero y fondos financieros y en muchas subculturas, el de ser agente de disciplina. Con el arribo y expansión del feminismo, los 
hombres se vieron presionados por la ideología así como la crítica de la sociedad a la participación activa y más directa en la crianza de los niños, y también en los quehaceres domésticos tales como la limpieza, el lavado o la cocina. Aunque actualmente no hay paridad de los roles en el hogar o en el trabajo, la participación masculina en el cuidado del niño aunque paulatina y esporádica, ha dado lugar al surgimiento de una vasta literatura al respecto y al progreso, aún lento de la revolución de roles en el hogar entre el hombre y la mujer (Berardo, Shehan y Leslie, 1987; Blair y Johnson, 1992).

La libertad reproductiva de la mijer que con ayuda de la tecnologia y el avance de las ciencias genéticas y de ingeniería humana, asi como la influencia ideológico/moral del feminismo proporciona una scrie de alternativas a la reproducción tradicional como la conocieron nuestros antepasados. Por ejemplo, la inseminación artificial, la búsqueda de madres sustitutas, inseminación de niños en vitro, entre otras formas reproductivas contemporáneas son alternativas que se debaten cotidianamente en hogares norteamericanos en adición a la adopción.

¿Cuál es el efecto con el niño y la familia de un origen tecnológico o de una niñez envuelta en cuestiones judiciales de paternidad o publicitarias? Aunque en minoría, varios de estos casos en Norteamerica promovieron serias controversias éticas y morales así como amplia publicidad y exposición periodística. Sin embargo, no se ha registrado estudios de investigación con respecto al impacto que estos inicios atípicos de vida tecnológica tienen en el desarrollo cognitivo-emocional o la identidad del niño. Aquí hay un campo amplio de investigación en los casos que aunque en la minoría, presentan no solamente un reto a la genética y la reproductiva tecnológica, sino también a la psicología del niño y las concepciones tradicionales del desarrollo emotivo y socio/ cognitivo heredadas de nuestras tradiciones cristiano/judeas occidentales.

Incremento de bogares no tradicionales o formas alternativas de familia. El feminismo y la ideología no sexista y no tradicionalista de la generación norteamericana contemporánea ha generado una diversidad de estilos de 
vida familiar en los que losniños crecen en hogares constituidos en múltiples y variadas formas. Desde el hogar tradicional con dos padres y hermanos biológicos a las formas de hogar con padres solteros (hombres o mujeres) que se han divorciado y han recibido la custodia por el juez o nunca se casaron y eligieron tener niños por adopción, la utilización de la inseminación artificial u otra alternativa, hogares formados por pareja homosexuales o formados de madrastras, padrastros y varias combinaciones de hijos o hermanastros. Los niños en Norteamérica están expuestos a una gran flexibilidad y heterogeneidad de estilos de vida que afectan la visión de la generación futura de la vida intima, conyugal y familiar. ¿Qué repercusiones tiene en el desarrollo emotivo/social y moral del niño, crecer en estos hogares no tradicionales, con alternativas tan diferentes $y$ combinaciones múltiples de paternidad, parentesco y vínculos familiares?

Las investigaciones científicas iniciadas en esta área se intensificarán en el próximo milenio desde que estas combinaciones familiares y los cambios demográficos representan un campo propicio a la investigación psicológica así como la exploración psico/educativa.

En resumen, la exposición emprendida en las páginas anteriores, revela que estos tres movimientos ideológico/culturales iniciados en la década de los cincuenta y continuados con fluctuaciones hasta el momento actual, han revolucionado la posición (o el planteamiento filosófico/ intelectual de la posición) de los marginados por raza, sexo y por la pobreza en la sociedad norteamericana.

Más allá de los resultados obtenidos, la ideología de estos tres movimientos: el feminista, de derechos civiles y de lucha contra la pobreza, ha generado un ímpetu por la paridad universal de aceptación y de derechos, sin paralelo en la historia contemporánea de este país. Este ímpetu ha generado también fuertes influencias centrífugas en varios países europeos y en otros en desarrollo. Estas influencias principalmente en Latinoamerica, se han expandido y asimilado en cada país de acuerdo a las necesidades oriundas de cada región, así como de acuerdo a la realidad sociohistórica nativa de cada pueblo. 


\section{Repercusiones para el Perú}

¿Cómo se puede aplicar este análisis de factores socio/culturales e ideológicos al estudio de nuestra psicología infantil latinoamericana y la del Perú en específico?

¿Cuáles son las repercusiones de los cambios ideológicos culturales internacionales (como el feminismo y el retorno de la mujer al trabajo en forma masiva) y de los movimientos subversivos político/sociales nacionales en la práctica y evolución de la psicología del niño/adolescente al iniciarse el nuevo milenio? y ¿Cómo es que las sociedades latinoame- ricanas pueden ser y son receptivas frente a las influencias del gigante norteño sin aún perder su individualidad, pero aún más, incorporándolas y adaptándolas con las tradiciones y el desenvolvimiento oriundo de cada pais?

Estas y otras más son reflexiones y cuestiones básicas de plantearse al enfocar un análisis de la psicología infantil en el Perú al arribo del nuevo siglo.

Podría decirse que de los tres movimientos ideológico/culturales referidos, es el feminismo y la liberación de la mujer en su expansión de roles y su incorporación al trabajo, lo que ha afectado más profundamente el panorama de la vida y la psicología infantil fuera de Norteamérica. El sexismo existente en el Perú siempre se debe enfocar desde el contexto cultural más amplio de la sociedad patriarcal norteamericana.

Indudablemente en el Perú, como en el resto de la economía global aún en culturas muy tradicionales, la mujer joven se ha incorporado con gran dinamismo a la producción económica ocasionando una convulsión interna de la vida doméstica y el cuidado de los niños. Sin embargo, en el Perú debido a la vigencia del sistema familiar extendido más común que el sistema nuclear (existente en Norteamérica), los familiares paternos o maternos, abuelos por su mayor parte, han asumido con más frecuencia el cuidado del niño. También el uso de niñeras o empleadas de servicio aunque 
no tan al alcance de todos como hace 20 años, es menos exclusivo que su uso en los EE.UU.

¿Qué otras diferencias y características propias pueden elucidarse al analizar si el retorno de la mujer/madre al trabajo en el Perú ha tenido un impacto en la educación, la crianza de los niños, la organización matrimonial, el índice de la reproducción? ¿Cómo es que estas influencias se han manifestado en zonas urbanas grandes en contraste con zonas rurales, en las que la madre indigena siempre ha trabajado fuera del hogar, por ejemplo? Debido a la caracteristica sobreprotectora de las madres en la cultura hispánica, ¿cuáles son los arreglos iniciados para cuidar al niño cuando la madre o padre está en el trabajo? ¿Existen arreglos típicos de nuestra sociedad? y icuáles son?

¿Existe un movimiento ideológico/político, indigenista y propiamente peruano que haya tenido un impacto en la evolución de nuestro conocimiento, acción o acercamiento psicológico al niño peruano? $\mathrm{La}$ aparición de las guerrillas como movimiento anárquico y subversivo en el Perú y su reino de terror durante los 1980 y parte de la década del 1990 puede considerarse como una erupción semejante en inspiración pero diferente en medios (los terroristas ilegales) al movimiento por lucha de derechos civiles e igualdad de los africanoamericanos en los Estados Unidos. ¿Qué impacto e influencia ha tenido el terrorismo y por ejemplo su política de reclutamiento de juventud en la educación, la práctica de la psicología y la composición o descomposición de la familia peruana?

El análisis exhaustivo de estos y otros fenómenos socio/políticos y culturales así como de factores sociodemográficos y legislativos en la realidad peruana, puede constituir una fuente poderosa y un impetu fuerte hacia el estudio de nuestra psicología infantil peruana en el momento actual, $y$ en sus perspectivas al inicio del nuevo siglo. 
La psicologia infantil

\section{Referencias}

Atkinson, R,C., y Shiffrin, R.M. (1977). Human memory: A proposed system and its control processes. En G.W. Bower (Ed.), Human memory: Basic processes. Nueva York: Academic Press.

Berardo, D.H., Shehan, C.L., y Leslie, G.R. (1987). A residue of tradition: Jobs, careers, and spouses time in housework. Joumal of Marriage and the Family, 49, 381-390.

Blair, S.L., y Johnson, M.P. (1992). Wives perceptions of the fairness of the division of household labor: The intersection of housework and ideology. Joumal of Marriage and the Family, 54, 570-581.

Bronfenbrenner, U. (1976). Reality and research in the ecology of human development. Master lectures on development psychology (Ms.1333). Washington, DC: American Psychological Association.

Cicirelli, V.G. (1969). The impact of Head Start: An evaluation of the effects of Head Start on children's cognitive and affective development. Washington, DC: National Bureau of Standards, Institute for Applied Technology.

Coleman, J.C., Hoffer, j., y Kilgore, s. (1966). Equality of educational opportunity. Washington, DC: U.S. Government Printing Office.

Committee for Economic Development. (1987). Children in need: Investment strategies for the educationally disadvantaged. Nueva York: Autor.

Dinnerstein, D. (1976). The mermaid and the minotaur: Sexual arrangements and buman malaise. Nueva York: Harper y Row.

Erikson, E. (1963). Childbood and society (2da. ed.). Nueva York: Norton. Flavell, J. (1977). Cognitive development. Englewood Cliffs, NJ: Prentice-Hall. Friedan, B. (1963). The feminine mystique. Nueva York: Dell Publishing Co. Inc.

Freud, S. (1952). A general introduction to psychoanalysis. Nueva York: Washington Square Press (Original work published 1920).

Gilligan, C.(1982). In a different voice: Psychological theory and women's development. Cambridge: Harvard University Press.

Halsell Miranda, A., y Santos de Barona, M. (1990). A model for interventions with low achieving minority students. En A. Barona y E. E. García (Eds.), Children at risk: Poverty, minority status, and other 
issues in educational equity (pp. 119-134). Washington, DC: National Association of School Psychologists.

Herrnstein, R.J., y Murray, C. (1994). Intelligence and class structure in American life. Nueva York: The Free Press.

Jensen, A.R. (1969). How much can we boost IQ and scholastic achievement? Harvard Educational Review,39, 1-123.

Kagan, J., y Moss, H.A. (1983). Birth to maturity: A study in psychological development. Cambridge: Harvard University Press.

Kohlberg, L. (1969). Stage and sequence: The cognitive developmental approach to socialization. En D.A. Goslin (Ed.), Handbook of socialization theory and research. Chicago: Rand McNally.

Lequerica, M. (1994a). Robert and Domenique: Report on family-centered and achild-centered intervention models applied at Head Start. InfantToddler Intervention: The Transdisciplinary Journal, 4, (1), 16-21.

Lequerica, M. (1994b). Stress and Hispanic children: A typology of assessment and intervention. New Jersey Psychologist,44 (1), 16-21.

Lewin, k. (1951). Field theory in social science: Selected theoretical papers (D. Cartwright, Ed.). Nueva York: Harper y Row.

Miller, G.A. (1962). Psychology: The science of mental life. Nueva York: Harper y Row.

Neisser, U. (1967). Cognitive Psychology. Englewood Cliffs, NJ: Prentice Hall.

Orfield, G. (1983). Public school desegregation in the United States 1968-1980. Washington, DC: Joint Center for Political Studies.

Peal, E., y Lambert, W. (1962). The relation of bilingualism to intelligence. Psychological Monographs, 76, 1-23.

Piaget, J. (1954). The construction of reality in the child (M. Cook, Trans.). Nueva York: Basic Books.

Piaget, J., e Inhelder, B. (1969). The psychology of the child (H. Weaver, Trans.). Nueva York: Basic Books.

Riley, D.A. (1977). Using local research to change 100 communities for children and families. American Psychologist, 52 (4), 424-433.

Rist, R.C. (1978). The invisible children: School integration in American society. Cambridge: Harvard University Press.

Robertson, 1. (1987). Sociology (3ra. ed.). Nueva York: Worth Publishers, Inc. 
Scarr, S. (1998). American child care today. American Psychologist, 53 (2), 95-108.

Schweinhart, L.J. (1985). Early childhood development programs in the eighties: The national picture. High Scope Early Childhood Policy Papers (No.1). Ypsilanti: High Scope Educational Research Foundation.

Skinner, B.F.(1951). Siience and buman bebavior. Nueva York: Macmillan. St. John, N. (1981). The effect of school desegration on children: A new look at the evidence. En A. Yarmolinsky et. Al. (Eds.), Race and schooling in the city. Cambridge: Harvard University Press.

Zigler, E. (1984). Meeting the critics on their own terms. American Psychologist, 39, 916-917.

Zigler, E. (1991). Using research to inform policy: The case of early intervention. En S.L. Kagan (Ed,), The case and education of America's young cbildren (pp. 154-172). Washington, DC: National Society for the Study of Education. 